\title{
Perception of global facial geometry is modulated through experience
}

Meike Ramon

Identification of personally familiar faces is highly efficient across various viewing conditions. While the presence of robust facial representations stored in memory is considered to aid this process, the mechanisms underlying invariant identification remain unclear. Two experiments tested the hypothesis that facial representations stored in memory are associated with differential perceptual processing of the overall facial geometry. Subjects who were personally familiar, or unfamiliar with the identities presented discriminated between stimuli whose overall facial geometry had been manipulated to maintain, or alter the original facial configuration (see Barton, Zhao \& Keenan, 2003). The results demonstrate that familiarity gives rise to more efficient processing of global facial geometry, and are interpreted in terms of increased holistic processing of facial information that is maintained across viewing distances. 
2 Author names and affiliations

3

4 Meike Ramon

5

6 Université catholique de Louvain

7 Institute of Research in Psychology

8 10, Place du Cardinal Mercier

$9 \quad$ B1348 Louvain-La-Neuve

10 Belgium

11 meike.ramon@uclouvain.be

12

13 University of Glasgow

14 Institute of Neuroscience and Psychology,

1558 Hillhead Street

16 G12QB Glasgow

17 United Kingdom

18 Meike.Ramon@glasgow.ac.uk

19 
Holistic face processing refers to the ability to simultaneously integrate facial information into a

21 unified percept (Sergent, 1984; Young, Hellawell \& Hay, 1987; McKone, Martini \& Nakayama, 2003;

22 Rossion, 2008). This ability, which other authors have coined configural, or face interactive processing

23 (Goffaux, 2009; for a review see e.g., Maurer, Le Grand \& Mondloch, 2002), is considered a hallmark

24 of human face processing expertise (Mondloch, Pathman, Maurer, Le Grand \& de Schonen, 2007).

25 Paradigms commonly used to measure holistic processing include the composite face illusion (Young

26 et al., 1987), the whole-part advantage (Tanaka \& Farah, 1993), or the face inversion effect (Yin, 1969),

27 all of which demonstrate the inter-dependency of perceiving information in upright faces (for recent

28 reviews see e.g., Rossion, 2009; 2013).

Another paradigm developed in the face processing literature that relies on holistic face

30 processing was reported by Barton, Zhao and Keenan (2003). They used faces of two unfamiliar

31 identities that were modified with respect to the different types of combinations of positional shifts of

32 facial features. In their oddity paradigm each trial involved simultaneous presentation of three face

33 stimuli that depicted one of these two unfamiliar identities. Two of the three stimuli were identical,

34 and subjects were required to identify the one that differed from them. Subjects' discrimination

35 performance was superior for combinations involving more severe distortions of the triangular

36 relation of the mouth and eyes (e.g. eyes closer and mouth down), as compared to those that

37 preserved the original aspect ratio (e.g. eyes farther and mouth down; see Figure 1 for an illustration).

38 In healthy observers this face geometry effect was found for upright, but not inverted face

39 discrimination. Moreover, the effect was not observed in the prosopagnosic patient TS, regardless of 
40 stimulus orientation. These findings were taken to reflect observers' ability to "integrate local spatial 41 information into overall facial structure".

We have previously reported similar findings in an investigation of personally familiar face 43 processing in pure prosopagnosia (Ramon \& Rossion, 2007; Ramon, Busigny, Gosselin \& Rossion, in 44 preparation). Specifically, we tested PS, an extensively studied pure case of prosopagnosia (first 45 reported by Rossion et al., 2003; see Rossion, 2014 for a recent review), and her colleagues who 46 worked together as kindergarten teachers. Adding to Barton et al.'s (2003) findings, we found that 47 healthy controls showed a face geometry effect during veridicality decisions between simultaneously 48 presented original and altered faces of the $\sim 30$ children they supervised. PS on the other hand did not 49 show this advantage for discrimination of more over less distorting changes of the overall facial 50 configuration.

These independent observations are to our knowledge the only studies having used this

52 paradigm. As both reported a lack of a face geometry effect in two cases of acquired prosopagnosia,

53 we reasoned that the manipulations used to measure the face geometry effect may represent an 54 alternative measure of holistic processing and face processing efficiency. However, subtle differences 55 between the aforementioned studies give rise to a number of questions.

First, are healthy observers with normal holistic face processing abilities equally capable of 57 discerning manipulations of facial geometry? The previous studies-using both unfamiliar and 58 personally familiar faces-indicate that this might indeed be the case. However, the reported 59 observations were made using two initially unfamiliar identities (Barton et al., 2003) presented 
60 repeatedly over a large number of trials, or a larger group of personally familiar ones encountered

61 repeatedly in real-life situations (Ramon \& Rossion, 2007; Ramon et al., in preparation). Consequently,

62 it is not clear whether discrimination of changes of the overall facial configuration, as well as the

63 observation of a face geometry effect require extensive (experimental, or real-life) exposure to the face

64 stimuli used. Moreover, it remains to be determined whether the observation of a face geometry effect

65 is confined to situations where stimuli are presented simultaneously.

The present study reports two experiments conducted to address the above questions, testing

67 subjects of an independent, larger cohort than previously (Ramon \& Rossion, 2007; Ramon et al., in

68 preparation). Here, using the same paradigm, the first experiment served to replicate the finding that

69 healthy observers can discern manipulations of the overall facial geometry, and show and advantage

70 for more as compared to less distorting ones (i.e., a face geometry effect) when comparing face stimuli

71 to internally stored facial representations. Observers who were personally familiar with the faces

72 depicted performed veridicality decisions between simultaneously presented original faces, paired

73 with altered versions that involved more or less distorting changes of the overall facial configuration.

74 In line with previous findings (Barton et al., 2003; Ramon \& Rossion, 2007; Ramon et al., in

75 preparation) we observed a face geometry effect, i.e. an advantage for discriminating between original

76 and more distorting changes of the facial configuration.

The second experiment sought to determine whether subjects' pre-experimentally acquired

78 familiarity with the identities would affect holistic processing-in terms of their discrimination

79 performance in general, and potentially the presence of a face geometry effect. To this end, familiar

80 and unfamiliar observers discriminated the same stimulus manipulations as in Experiment 1 in the 
81 context of a delayed matching task. This was chosen deliberately as simultaneous stimulus

82 presentation of unfamiliar faces for long durations encourage an image, or feature-by-feature

83 matching strategy - the antidote of holistic processing, which operates in particular given short

84 exposure durations (e.g., Davidoff, 1986; Hole, 1994; Stollhoff, Jost, Elze \& Kennerknecht, 2010). Here

85 only familiar subjects were able to perceive differences in changes of the overall facial configuration

86 (as evidenced by their above chance level performance, which controls did not exceed), but did not

87 exhibit a face geometry effect. Together, these results indicate that personal familiarity is associated

88 with more efficient integration of the overall facial configuration, i.e. holistic processing, but that the

89 observation of a face geometry effect may depend on stimuli being presented simultaneously.

\section{Materials and Methods}

The personally familiar faces from which stimuli were created here were comparably familiar to

92 all individuals of the peer groups tested (senior year psychology master students from the University

93 of Louvain). This procedure has been applied elsewhere (Ramon, in press) and bears the advantage of

94 having stimulus sets of personally familiar faces, that are identical across subjects, and larger in size

95 (here: 26 and 14 identities in Experiments 1 and 2, respectively) than those utilized in studies using

96 subject-specific sets of personally familiar faces (e.g. two (Arsalidou, Barbeau, Bayless \& Taylor, 2010)

97 to six (Gobbini et al., 2013) personally familiar identities). Control subjects tested in Experiment 2

98 were psychology students from a different cohort who were unfamiliar with the identities depicted;

99 all subjects received financial compensation for participation. The experiments were undertaken with

100 the understanding and written consent of each subject, and conform to The Code of Ethics of the 
101 World Medical Association (Declaration of Helsinki). Consent for publication was obtained for 102 individuals depicted in the figures exemplifying stimuli used.

103 Despite offering more control of the stimulus material (all images were taken under identical

104 conditions by the experimenter, as opposed to being provided by subjects; e.g. Gobbini et al., 2013),

105 and enabling larger numbers of trials (with comparably less image repetitions), the approach using

106 faces derived from the same cohort comes at the expense of a limited number of available potential

107 participants. As previously (Ramon et al., 2011; Ramon, in press), classical parametric statistical

108 methods were complemented with robust techniques (percentile bootstrap analyses). Given their

109 higher statistical power and robustness to deviations from the assumed optimal distribution

110 parameters, the latter are particularly recommended given small sample sizes and/or unknown

111 theoretical distributions of a statistic of interest (Adèr, Mellenbergh \& Hand, 2008). To investigate

112 performance differences related to the experimental manipulations, for each behavioral measure

113 subjects were sampled with replacement and differences between the bootstrap populations for the

114 conditions in question were computed. This process was repeated 999 times, leading to a distribution

115 of bootstrapped estimates of the mean differences across conditions. Differences between the sample

116 means were considered significant if the $95 \%$ bootstrap confidence intervals (btCIs) did not include

117 zero. Relying on an estimation of the $\mathrm{H}_{1}$, this bootstrap technique tends to have more power than

118 other robust methods that evaluate the null hypothesis (Wilcox, 2012). Across experiments, these

119 analyses were conducted on accuracy scores (proportion correct) and correct reaction times (RTs);

120 effect sizes are provided where differences reached significance. 
121 Experiment 1: Veridicality decisions for personally familiar faces across manipulations of overall

122 facial geometry

Participants. Thirteen subjects who were personally familiar with the faces presented as stimuli

124 (mean age: 24 \pm ; eight female; one left-handed male) participated in Experiment 2.

Stimuli. Full frontal color photographs of 26 students were cropped of hair and external

126 features using Adobe Photoshop. The resulting stimuli (originals; 154-183 pixels wide, 218-256 pixels

127 high) were modified to create four altered face stimuli per identity. The inter-ocular and nose-mouth

128 distances could be either increased or decreased, with changes always applied in conjunction. That is,

129 the eyes were moved either out (EO) or in (EI), and the mouth was moved up (MU) or down (MD),

130 giving rise to one image per experimental condition (EO_MU, EI_MD, EI_MU, EO_MD) per identity.

131 Given the size of the stimuli, the features were displaced by two pixels per feature. The stimulus

132 modifications applied to each original face are illustrated in Figure 1.

Apparatus and procedure. Stimuli were displayed using Eprime software, on a 19" monitor

134 (58cm viewing distance, $1280 \times 1024$ pixel resolution). Stimulus (i.e., face) size comprised 4.5-5.2 ${ }^{\circ}$

135 (width) and $6.5-7.5^{\circ}$ (height) of visual angle (VA), respectively. Participants performed a veridicality

136 decision task; they decided which of two juxtaposed stimuli (presented until response provided)

137 displayed the original face of the identity depicted on a given trial by pressing a left or right key,

138 respectively. The experiment consisted of four blocks of equal length with interleaved pauses. In total

139 participants completed 208 trials (26 identities $x$ four change types $x$ two possible locations for

140 original faces), which were separated by a 1000ms inter-trial interval and randomly assigned to the 
141 four blocks (trial presentation randomized). Prior to the actual experiment, participants completed

142 four practice trials (excluded from analysis).

143 Experiment 2: Delayed matching across manipulations of overall facial geometry for personally 144 familiar and unfamiliar faces

145 Participants. Two groups of twelve subjects each were tested in Experiment 2. Participants of

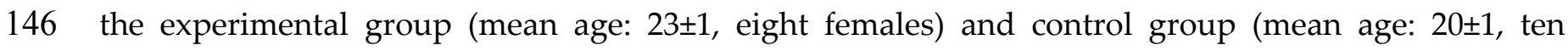
147 females) differed only with respect to their personal familiarity with the identities presented as 148 stimuli (familiar, unfamiliar).

Stimuli. A subset of stimuli created for Experiment 1 was used in Experiment 2.; this subset

150 included original (i.e., veridical) faces of 14 individuals and their respective modified versions (see

151 Figure 1). This smaller stimulus set was chosen given the different procedure and number of trials

152 necessary (see below). As here the stimuli were presented individually, and thus could be presented

153 at a larger size (between 185 and 218 pixels in width, and 260 and 307 pixels in height due to inter-

154 individual differences in face size and shape), feature displacements amounted to $\sim 5$ pixels per feature

155 for each of the four possible modified versions of a face.

157 (58cm viewing distance, $1280 \times 1024$ pixel resolution). Probe and test faces' height comprised 5.4-6.3

158 (width) and 7.8-9.0 (height) of VA, respectively. Participants performed a delayed matching to 159 sample task, requiring same/different decisions between the sample and subsequently presented 160 probe stimulus on a given trial by pressing a left or right key, respectively. 
162 from the analyses) throughout which two female identities and their respective altered versions were

163 presented. Subjects were prompted to be as accurate as possible and attempt to achieve over $80 \%$

164 correct. The two blocks differed in that for the first, feedback was provided on each individual trial,

165 while during the second block feedback was provided after the first and second half of the practice

166 block. After both practice blocks the total score was provided and participants were again encouraged

167 to perform as accurately as possible and maintain high scores.

170 fixation cross was presented for $400 \mathrm{~ms}$, followed by a $200 \mathrm{~ms}$ blank and a sample stimulus displayed

171 for 500ms. After a 600ms ISI, a probe stimulus was presented for a maximum of 3000ms during which

172 responses were recorded. Consecutive trials were spaced by a 900ms inter-trial interval; the positions

173 of sample and probes were jittered randomly to avoid participants adopting local matching strategies.

174 Trials required to investigate the research question posed in Experiment 2 were those for 175 which sample and probe stimulus pairs comprised the original face with each altered version 176 (EO_MU, EI_MU,EO_MD,EI_MD). In total there were 96 of these (original/altered) trials of interest:

17712 identities $x$ four pairs $x$ two orders (original probe followed by altered sample, or vice versa). An 178 additional 96 trials, also requiring a "different" response, were included which involved presentation 179 of two altered versions as samples and probes (altered/altered). These trials were included to avoid 180 subjects performing their decisions by focusing on a single facial region. Altered/altered trials were 181 chosen so that each of the six possible combinations occurred at least once per identity; an additional 
182 two trials per identity was chosen pseudo-randomly ensuring that overall all combinations occurred

183 with equal frequency. Note that performance on these catch trials could not be distinguished in terms

184 of "more" or "less" distorting changes, as the consecutively presented stimuli sometimes involved a

185 single change (eyes or mouth, e.g. if $E O_{-} M D$ and $E O \_M U$ were presented in succession) or

186 combinations of changes (both eyes and mouth, e.g. if EO_MD and EI_MU were presented

187 consecutively). ${ }^{1}$ A further 192 trials were included which involved stimulus repetition, i.e., required

188 "same" responses. For each of the 12 identities these "same" trials involved a minimum of three

189 repetitions for each of the five possible stimulus types (i.e. original, EO_MU, EI_MU, EO_MD, EI_MD);

190 an additional trial per identity was chosen pseudo-randomly ensuring that overall each stimulus type

191 was repeated with equal frequency. Note that the catch (altered/altered) and "same" trials were

192 included in order to ensure equal amounts of trials requiring same and different responses, and

193 identify potentially insufficiently high performance for the trials of interest.

195 combinations used across subjects), with interleaved pauses every 64 (randomly presented) trials,

196 after which feedback on performance was provided. The presence of a face geometry effect was

197 investigated based on, and provided sufficiently high discrimination performance for the 96 trials of

198 interest involving consecutive presentation of original and altered facial configurations.

\footnotetext{
${ }^{1}$ Investigating the effect of single inter-feature relational changes as a function of region, or as compared to combined changes on discrimination performance is an interesting research question in itself (see e.g. Barton et al., 2003; Malcolm, Leung \& Barton, 2004). However, this was not the research question addressed here; each of these conditions was represented by fewer trials, as compared to the trials of interest (original/altered), therefore making direct comparisons impossible.
} 
200 Experiment 1: Veridicality decisions for personally familiar faces across manipulations of overall 201 facial geometry

203 discriminated more readily than others in personally familiar faces. Note that other authors have used 204 the term 'facial configuration' to refer to single distances between individual features (Maurer et al., 205 2002), or include other facial information (e.g. face contour; Sergent, 1984; Young et al., 1987). Here 206 'facial configuration' refers to the spatial relationship between the most diagnostic internal facial 207 features - the eyes/eyebrows and mouth (see e.g. Haig, 1985; Gosselin \& Schyns, 2001; Sadr, Jarudi \& 208 Sinha, 2003) - using stimulus manipulations originally introduced by Barton et al. (2003). Participants 209 who were personally familiar with the individuals depicted performed identity veridicality decisions 210 for image pairs comprising the original face, along with one that was modified with respect to its 211 facial configuration (see Figure 1). In line with previous findings (Barton et al., 2003; Ramon \& 212 Rossion, 2007; Ramon et al., in preparation), modifications that alter the original facial configuration 213 (i.e., are more distorting) were hypothesized to be more readily perceived than those that preserve it 214 (i.e., are less distorting).

215 Figure 2a displays the group's mean performance for more and less distorting manipulations 216 of the overall facial geometry. The planned comparison yielded that more distorting changes were 217 more readily rejected than less distorting ones (accuracy: $t(12)=1.49, p=.16$; RTs: $t(12)=-3.04, p=.01$, $\left.218 r^{2}=.44\right)$. Percentile bootstrapped estimates of the mean difference between distortion types (i.e., more 
219 and less distorting alterations of the overall facial geometry) were computed to validate the distortion-

220 related differences in performance observed with canonical statistics. This analysis confirmed the

221 pattern of superior performance for trials on which original faces were presented together with more,

222 as opposed to less distorting versions (btCIs for all measures: accuracy: [-.002, .05]; RTs: [-474, -121]).

223 While the non-significant trends observed for accuracy scores as tested parametrically could be

224 related to the distribution of the data, the results provided by robust statistics confirm the absence of

225 an effect of condition for this behavioral measure (see also Figure $2 b$ and $3 c$ for differences in number

226 of errors and RT across conditions for individual subjects). Thus, canonical and robust statistics

227 confirmed the hypothesis that more distorting manipulations were discriminated more readily from

228 original faces than less distorting ones.

229 Experiment 2: Delayed matching across manipulations of overall facial geometry for personally

230 familiar and unfamiliar faces

231 Experiment 1 demonstrated an advantage for detecting more over less distorting alterations of

232 the overall facial geometry, i.e., a face geometry effect, when subjects made veridicality decisions

233 between original and altered faces of personally familiar individuals. Following this observation, in

234 Experiment 2 two groups of observers performed a delayed matching task, which did not require face

235 identification, but merely same/different decisions between consecutively presented stimuli.

236 Performance for discriminating changes in the overall facial geometry was considered an index of

237 holistic processing, the hypothesis being that personal familiarity with faces leads to increased

238 integration of facial information from across the entire face, manifesting in terms of more efficient

239 perception of global facial geometry, and potentially a face geometry effect. 
Although only trials involving original/altered faces were relevant to investigate the

241 emergence of a face geometry effect, subjects' accuracy for catch trials was inspected to ensure

242 sufficient performance. Figure 3a displays the groups' mean performance for trials of interest and

243 both types of catch trials. Independent sample t-Tests revealed no differences in groups' performance

244 for catch trials involving stimulus repetition, $t(22)=.94$, $n s$, or presentation of two altered versions,

$245 \mathrm{t}(22)=.86$, ns. However, for the trials of interest, i.e., stimulus pairs involving original and altered facial

246 configurations, familiar subjects performed significantly better than unfamiliar controls, $t(22)=3.50$,

$247 p=.001$. A $X^{2}$ test of proportions revealed that controls' performance did not differ significantly from

248 chance $(p=.24)$, contrary to that of familiar subjects' $(p<.02)$. Although on only familiar subjects' data

249 were therefore further analyzed for potential face geometry effects, unfamiliar subjects' performance

250 for more, as compared to less distorting trials is displayed in Figure 3b.

252 distorting changes (accuracy: $t(11)=-.77$; RTs: $t(11)=-1.72$; both $n s$ ). Percentile bootstrapped estimates of

253 the mean difference between distortion types (i.e., more and less distorting alterations of overall facial

254 geometry) confirmed the parametrically observed lack of a difference between less and more 255 distorting conditions (btCIs for all measures: accuracy: [-.07, .02]; RTs: [-88, 3]). Individual subjects' 256 differences in number of errors and RT across conditions are displayed in Figure 3b and 3c.

\section{General Discussion}

The aim of the present paper was to determine whether the presence of a facial representation in

259 memory, as for personally familiar faces, would lead to differences in holistic processing, which is 
260 considered a hallmark of face processing. Contrary to the commonly used paradigms involving 261 combinations of facial information from different identities (Young et al., 1987; Tanaka \& Farah, 1993),

262 or displacements of individual inter-feature distances (Goffaux \& Rossion, 2007; Sekunova \& Barton, 263 2008; Ramon \& Rossion, 2010), here a novel paradigm involving manipulations of the overall facial 264 geometry (Barton et al., 2003) was applied. The experiments reported were rooted in the idea that 265 repeated real life exposure with personally familiar faces would give rise to differential processing of 266 global facial geometry.

Barton et al.'s (2003) seminal experiment involved an oddity paradigm, in which observers were required to indicate which of three simultaneously presented unfamiliar face stimuli was different

269 from the remaining (identical) two. In their experiment various manipulations of facial information, 270 including changes of feature color, or single inter-feature distances, were discriminated. Additionally, 271 the overall facial configuration was also manipulated via combinations of altered inter-feature 272 distances between the most diagnostic facial features: the eyes and mouth (Haig, 1985; Gosselin \& 273 Schyns, 2001; Sadr et al., 2003). Beyond finding more efficient discrimination for the latter, the authors 274 reported that specific changes to the overall facial configuration were more readily discerned: those 275 that distorted, as opposed to maintained the original feature configuration. Importantly, this was 276 observed only in healthy controls, but not in a case of acquired prosopagnosia.

277 Experiment 1 involved simultaneous veridicality decisions between the original face of 278 personally familiar individuals and a modified version, in which the overall configuration had been 279 altered. Replicating our previous findings under identical experimental conditions (Ramon \& Rossion, 280 2007; Ramon et al., in preparation), subjects showed a face geometry effect for correct RTs-i.e. 
281 superiority for discriminating more, as compared to less distorted versions of the original inter282 feature ratios (Figure 2). The lack of a significant effect for accuracy scores, as reported by Barton et al. 283 (2003) may be related to the difference in paradigms and tasks applied. In Barton et al.'s (2003) study, 284 subjects had to identify the 'odd' one out of a set of three face stimuli. Here, however, subjects had to 285 identify the veridical face of one of their classmates that was presented together with its more or less 286 distorting version. Comparable accuracy scores across conditions were achieved at the expense of 287 prolonged RTs when trials involved less distorting changes of the overall facial configuration. That is, 288 faces presented on these trials required more visual inspection to be correctly reject foils as deviating 289 from the facial representation stored in memory. As all features were displaced independently, 290 perception of only the eye, or mouth location would not have led to this observed benefit for more, 291 over less distorting changes of the facial configuration. The observation of this face geometry effect is 292 therefore interpreted in terms of subjects perceiving both sources of information simultaneously, i.e. 293 integrating information from across the entire face.

Experiment 2 sought to determine whether personal familiarity would be associated with an advantage for processing the overall facial geometry in the context of a delayed matching task, which 296 could be completed by unfamiliar subjects as well. Theoretically, performance on this task did not 297 require a facial representation stored in memory, and could be achieved based on matching of the 298 sequentially presented visual input. First we assessed whether familiar and unfamiliar subjects could 299 generally discriminate changes of the overall facial configuration (i.e., irrespective of the type of 300 change). Provided sufficiently high performance, their behavior was then analyzed to determine 301 whether they exhibit a face geometry effect as found in Experiment 1. Two interesting observations 
were made in Experiment 2. First, only familiar observers were able to detect the presence of a

303 difference in face pairs consisting of original and altered versions, while unfamiliar observers

304 performed at chance (Figure 3a). As both the inter-ocular and nose/mouth distances had to be considered simultaneously, controls' insufficient performance indicates that they were not capable of doing so. Secondly, no face geometry effect was observed in familiar observers (Figure 3b). That is, under sequential matching conditions, the presence of a facial representation stored in memory led to enhanced face discrimination performance, but not the observation of a face geometry effect. (2003): contrary to familiar subjects, our unfamiliar healthy observers were not able to discern

311 manipulations of the overall facial geometry. However, two related aspects might account for these 312 seemingly conflicting findings. First, despite using a comparable number of trials, Barton et al.'s 313 (2003) stimuli depicted only two identities, leading to a larger degree of experimental familiarization

314 with their unfamiliar face stimuli, which may have facilitated discrimination performance. Moreover, 315 the unfamiliar faces used here were "subjected to judgments of sameness and difference" (Sergent, 316 19984). This was done in the context of a delayed matching task involving sequential, as opposed to 317 simultaneous stimulus presentation. The introduction of a delay interval between the presentation of 318 probe and sample faces has been associated with a decay of the inner features (e.g. Rock, Halper \& 319 Clayton, 1972; Walker-Smith, 1978). This delay is likely to have increased task difficulty for 320 discrimination of subtle changes of the facial configuration applied to the same identity. 
323 for controls' inability to discern changes of the overall facial configuration. Familiar subjects' ability to

324 detect these changes on the other hand indicates that the presence of a facial representation in 325 memory minimizes the aforementioned information decay related to the inter-stimulus delay. The 326 results of Experiment 2 therefore suggest that familiar observers, which exhibit a face geometry effect 327 given simultaneous presentation (Experiment 1; Ramon \& Rossion, 2007; Ramon et al., in 328 preparation), can extract information concerning the overall facial geometry given short presentation 329 durations and maintain it more efficiently than unfamiliar subjects, who lack facial representations 330 stored in memory. As stated above, personal familiarity was expected to lead "to increased 331 integration of facial information from across the entire face, manifesting in terms of more efficient 332 perception of global facial geometry". Since discrimination of these changes-even in the absence of a

333 face geometry effect-relies on observers' ability to "integrate local spatial information into overall 334 facial structure" (Barton et al., 2003), personally familiar subjects exhibited greater holistic processing, 335 i.e. an increased perceptual field of view (see also Rossion, 2008; Rossion, 2009; Van Belle, Lefevre \& 336 Rossion, 2015) than unfamiliar controls.

An open question the present study cannot answer concerns which aspects of real-life 338 familiarity lead to this increased processing efficiency. Previous research suggests that processing of 339 personally familiar faces calls upon different processes than those involved in recognition of famous 340 or experimentally familiarized faces (Tong and Nakayama 1999; Knappmeyer, Thornton \& Bülthoff, 341 2003; Carbon, 2008). One main difference between personally familiar and unfamiliar, or 342 experimentally learned faces, concerns the degree of variability in the visual input at encoding or 343 learning phases. Experimentally acquired familiarity typically involves image learning (e.g. Caldara et 
344 al., 2005; Tanaka, Curran, Porterfield \& Collins, 2006; Herzmann \& Sommer, 2007; 2010; Barsics \&

345 Bredart, 2012), and/or a restricted number of different images per identity (O'Donnell \& Bruce, 2001;

346 Gobbini \& Haxby, 2006; Tanaka et al., 2006), most commonly presented with constant stimulus size.

347 Personally familiar faces on the other hand are encountered across a range of viewing conditions,

348 rendering their representations and therefore identification robust to variations including changes in

349 viewpoint and exposure across different viewing distances. With varied viewing distance the visual

350 information projected to the retina changes, leading to differences in the available spatial frequency

351 content (Sowden \& Schyns, 2006). As demonstrated in Figure 4, personally familiar faces can be

352 reliably identified despite large variations of available information associated with changes in viewing

353 distances, and are processed with higher efficiency than their unfamiliar counterparts (Kemp, Towell

354 \& Pike, 1997; Bruce et al., 1999).

355 These visual input variations during face learning in real-life scenarios may form the basis for

356 familiarity-dependent differential processing manifesting in terms of prioritized detection or

357 increased behavioral recognition speed (Herzmann, Schweinberger, Sommer \& Jentzsch, 2004;

358 Ramon, Caharel \& Rossion, 2011; Gobbini et al., 2013). They may also account for familiarity-related

359 differences in perceptual processing suggested by studies of oculo-motor patterns (Van Belle, Ramon,

360 Lefèvre \& Rossion, 2010), discrimination of feature displacements (Brooks \& Kemp, 2007), and spatial

361 frequency thresholds (Watier \& Collin, 2009). Importantly, changes in viewing distances lead to

362 perception of an individual's facial configuration across a range of spatial frequencies, which may be

363 responsible for the increased discrimination sensitivity observed here, and familiarity-dependent

364 differences in processing of vertical inter-feature distances (Ramon, in press). 
365 Further studies are required to address this assumption, ideally involving larger sample sizes to

366 detect potentially small effects. Moreover, a longitudinal approach would be favorable in order to e.g.

367 track the development of participants' sensitivity to the overall facial configuration throughout the

368 course of familiarization. A further necessary aspect such studies should consider is the systematic

369 control of visual input variations, e.g. viewing distances (Sowden \& Schyns, 2006), facial motion

370 (Knappmeyer et al., 2003) and viewpoint changes (Stevenage \& Osborne, 2006) throughout the course

371 of familiarity acquisition.

372 The present results expand on previous neuropsychological findings that demonstrate the 373 importance of holistic processing for perception of overall facial geometry (Barton et al., 2003; Ramon 374 \& Rossion, 2007; Ramon et al., in preparation). They are interpreted as evidence that personal

375 familiarity with faces is associated with increased sensitivity to the overall facial configuration, i.e., 376 holistic processing. Taken together, the results reported here indicate that holistic processing is 377 facilitated by the presence of a facial representation stored in memory.

Aknowledgements

The author expresses her gratitude to Bruno Rossion, under the supervision of which the 380 experiments were carried out. Further thanks are directed to Philippe Schyns and Luca Vizioli, to all 381 participants for their cooperation, as well as two anonymous reviewers for their helpful comments on 382 an earlier version of this paper. 


\section{References}

384 Adèr HJ, Mellenbergh GJ \& Hand DJ (2008). Advising on research methods: A consultant's 385 companion. Huizen, The Netherlands: Johannes van Kessel Publishing.

386 Arsalidou M, Barbeau EJ, Bayless SJ \& Taylor MJ (2010). Brain responses differ to faces of mothers and 387 fathers. Brain Cogn, 74(1):47-51.

388 Barsics C \& Brédart S (2012). Recalling semantic information about newly learned faces and voices.

389 Memory, 20, 527-34.

390 Barton JJS, Zhao J \& Keenan JP (2003). Perception of global facial geometry in the inversion effect and 391 prosopagnosia. Neuropsychologia, 41, 1703-1711.

392 Brooks KR \& Kemp RI (2007). Sensitivity to feature displacement in familiar and unfamiliar faces:

393 beyond the internal/external feature distinction. Perception, 36, 1646-1659.

394 Bruce V, Henderson Z, Greenwood K, Hancock P, Burton M \& Miller P (1999). Verification of face

395 identities from images captured on video. J Exp Psychol Appl, 5, 339-360.

396 Burt PJ \& Adelson EH (1983). The Laplacian pyramid as a compact image code. IEEE Trans Commun, $39731,532-540$.

398 Caldara R, Schyns P, Mayer E, Smith ML, Gosselin F \& Rossion B (2005). Does prosopagnosia take the 399 eyes out of face representations? Evidence for a defect in representing diagnostic facial information 400 following brain damage. J Cogn Neurosci, 17, 1652-66.

401 Carbon CC (2008). Famous faces as icons. The illusion of being an expert in the recognition of famous 402 faces. Perception, 37, 801-806. 
403 Gobbini MI \& Haxby JV (2006). Neural response to the visual familiarity of faces. Brain Res Bull, 71, 404 76-82.

405 Gobbini MI, Gors JD, Halchenko YO, Rogers C, Guntupalli JS, Hughes H \& Cipolli C (2013). 406 Prioritized Detection of Personally Familiar Faces. PLoS One, 21;8(6):e66620.

407 Goffaux V \& Rossion B (2007). Face inversion disproportionately impairs the perception of vertical but 408 not horizontal relations between features. J Exp Psychol Hum Percept Perform, 33, 995-1002.

409 Goffaux V (2009). Spatial interactions in upright and inverted faces: re-exploration of spatial scale 410 influence. Vision Res, 49, 774-81.

411 Gosselin F \& Schyns PG (2001). Bubbles: a technique to reveal the use of information in recognition 412 tasks. Vision Res, 41, 2261-2271.

413 Haig ND (1985). How faces differ--a new comparative technique. Perception, 14, 601-615.

414 Herzmann G \& Sommer W (2007). Memory-related ERP components for experimentally learned faces 415 and names: characteristics and parallel-test reliabilities'. Psychophysiology, 44, 262-276.

416 Herzmann G \& Sommer W (2010). Effects of previous experience and associated knowledge on 417 retrieval processes of faces: an ERP investigation of newly learned faces. Brain Res, 14, 54-72.

418 Herzmann G, Schweinberger SR, Sommer W \& Jentzsch I (2004). What's special about personally 419 familiar faces? A multimodal approach. Psychophysiology, 41, 688-701.

420 Hole GJ (1994). Configurational factors in the perception of unfamiliar faces. Perception, 23, 65-74.

421 Kemp R, Towell N \& Pike G (1997). When seeing should not be believing: Photographs, credit cards 422 and fraud. Appl Cogn Psychol, 11, 211-222.

423 Knappmeyer B, Thornton IM \& Bülthoff HH (2003). The use of facial motion and facial form during 424 the processing of identity. Vision Research, 43, 1921-1936. 
425 Loftus GR \& Harley EM (2004). How different spatial-frequency components contribute to visual

426 information acquisition. J Exp Psychol Hum Percept Perform, 30, 104-18.

427 Malcolm GL, Leung C, Barton JJ. Regional variation in the inversion effect for faces: differential effects

428 for feature shape, feature configuration, and external contour. Perception. 2004;33(10):1221-31.

429 Maurer, D., Le Grand, R., \& Mondloch, C.J. (2002). The many faces of configural processing. Trends

430 Cogn Sci, 6, 255-260.

431 McKone, E., Martini, P., \& Nakayama, K. (2003). Isolating holistic processing in faces (and perhaps

432 objects). In M. Peterson \& G. Rhodes (Eds.), Perception of faces, objects, and scenes: Analytic and 433 holistic processes (pp. 92-119). Oxford, UK: Oxford University Press.

434 Mondloch C, Pathman T, Maurer D, Le Grand, R \& de Schonen S (2007). The composite face effect in 435 six-year-old children: Evidence of adult-like holistic face processing. Visual Cognition, 15, 564-577.

436 O'Donnell C \& Bruce V (2001). Familiarisation with faces selectively enhances sensitivity to changes 437 made to the eyes. Perception, 30, 755-764.

438 Ramon M \& Rossion B (2010). Impaired processing of relative distances between features and of the 439 eye region in acquired prosopagnosia--two sides of the same holistic coin? Cortex, 46, 374-89.

440 Ramon M (in press). Differential processing of vertical inter-feature relations due to real-life 441 experience with personally familiar faces. Perception.

442 Ramon M, Busigny T, Gosselin G \& Rossion B (in preparation). All new kids on the block? Personally 443 familiar face processing in a kindergarten teacher with pure prosopagnosia.

444 Ramon M, Caharel S \& Rossion B (2011). The speed of recognition of personally familiar faces. 445 Perception, 40, 437-449. 
446 Ramon, M., \& Rossion, B. (2007). What's lost in prosopagnosia? An investigation of familiar face 447 processing in a single-case of pure prosopagnosia working in a kindergarten [Abstract]. J Vis, 7, 122a.

448 Rock I, Halper F \& Clayton T (1972). The perception and recognition of complex figures. Cognitive 449 Psychology, 3, 655-673.

450 Rossion B (2009). Distinguishing the cause and consequence of face inversion: the perceptual field 451 hypothesis. Acta Psychol, 132, 300-12.

452 Rossion B (2013). The composite face illusion: a whole window into our understanding of holistic face 453 perception. Visual Cognition, 21, 139-253.

454 Rossion B (2014). Understanding face perception by means of prosopagnosia and neuroimaging. Front 455 Biosci (Elite Ed), Jun 1;6:258-307.

456 Rossion B, Caldara R, Seghier M, Schuller AM, Lazeyras F \& Mayer E (2003). A network of occipito457 temporal face-sensitive areas besides the right middle fusiform gyrus is necessary for normal face 458 processing. Brain, 126, 2381-95.

459 Rossion, B. (2008). Picture-plane inversion leads to qualitative changes of face perception. Acta 460 Psychologica, 128, 274-289.

461 Sadr J, Jarudi I \& Sinha, P (2003). The role of eyebrows in face recognition. Perception, 32, 285-293.

462 Sekunova A \& Barton JJ (2008). The effects of face inversion on the perception of long-range and local 463 spatial relations in eye and mouth configuration. J Exp Psychol Hum Percept Perform, 34, 1129-35.

464 Sergent J (1984). Configural processing of faces in the left and the right cerebral hemispheres. J Exp 465 Psychol Hum Percept Perform, 10, 554-572.

466 Smith FW \& Schyns PG (2009). Smile through your fear and sadness: transmitting and identifying 467 facial expression signals over a range of viewing distances. Psychol Sci, 20, 1202-8. 
468 Sowden PT \& Schyns PG (2006). Channel surfing in the visual brain. Trends Cogn Sci, 10, 538-45.

469 Stevenage, Sarah V. and Osborne, Cara D. (2006) Making heads turn: the effect of familiarity and

470 stimulus rotation on a gender-classification task. Perception, 35, 1485-1494.

471 Stollhoff R, Jost J, Elze T \& Kennerknecht I (2010). The early time course of compensatory face

472 processing in congenital prosopagnosia. PLoS One, 5(7):e11482.

473 Tanaka JW \& Farah MJ (1993). Parts and wholes in face recognition. Q J Exp Psychol A, 46, 225-245.

474 Tanaka JW, Curran T, Porterfield AL \& Collins D (2006). Activation of preexisting and acquired face 475 representations: the N250 event-related potential as an index of face familiarity. J Cogn Neurosci, 18, 476 1488-1497.

477 Tong F \& Nakayama K (1999). Robust representations for faces: evidence from visual search. J Exp 478 Psychol Hum Percept Perform, 25, 1016-1035.

479 Van Belle G, Lefèvre P, Rossion B (2015). Face inversion and acquired prosopagnosia reduce the size 480 of the perceptual field of view. Cognition, 31, 403-408.

481 Van Belle G, Ramon M, Lefèvre P \& Rossion B (2010). Fixation patterns during recognition of 482 personally familiar and unfamiliar faces. Front Psychology 1:20. doi:10.3389/fpsyg.2010.00020.

483 Walker-Smith GJ (1978). The effects of delay and exposure duration in a face recognition task. 484 Perception \& Psychophysics, 24, 63-70.

485 Watier NN \& Collin CA (2009). Effects of familiarity on spatial frequency thresholds for face 486 matching. Perception, 38, 1497-507.

487 Wilcox RR (2012). Introduction to robust estimation and hypothesis testing (2nd ed.). San Diego: 488 Academic Press.

489 Yin RK (1969). Looking at upside-down faces. Journal of Experimental Psychology, 81, 141-145. 
490 Young, AW, Hellawell, D \& Hay DC (1987). Configurational information in face perception.

491 Perception, 16, 747-759. 


\section{1}

Examples of stimuli used in Experiments 1 and 2.

Examples of stimuli used in Experiments 1 and 2. Stimulus manipulations included combined changes of the inter-ocular and nose-mouth distance, giving rise to the four possible combinations depicted here. Two types of changes were considered as "less" (black borders), or "more" distorting (gray borders), as the eyes and mouth were moved either in the same (eyes/mouth out, eyes/mouth in) or opposite (eyes out/mouth in, eyes in/mouth out) direction, thereby respectively preserving, or altering the original facial configuration.

\section{Eyes out}

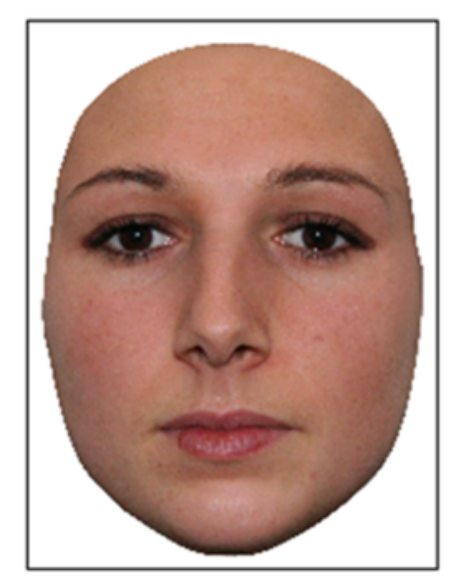

\section{Original face}

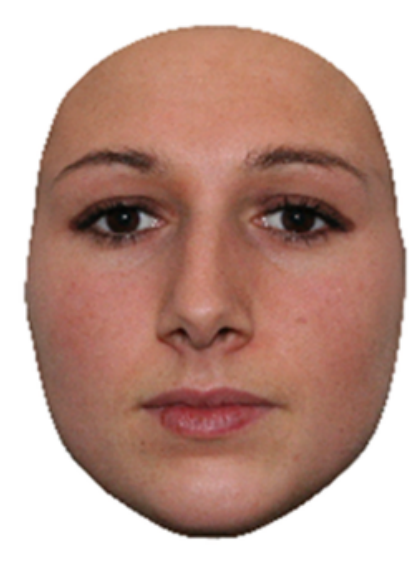

Mouth down

Mouth up
Eyes in
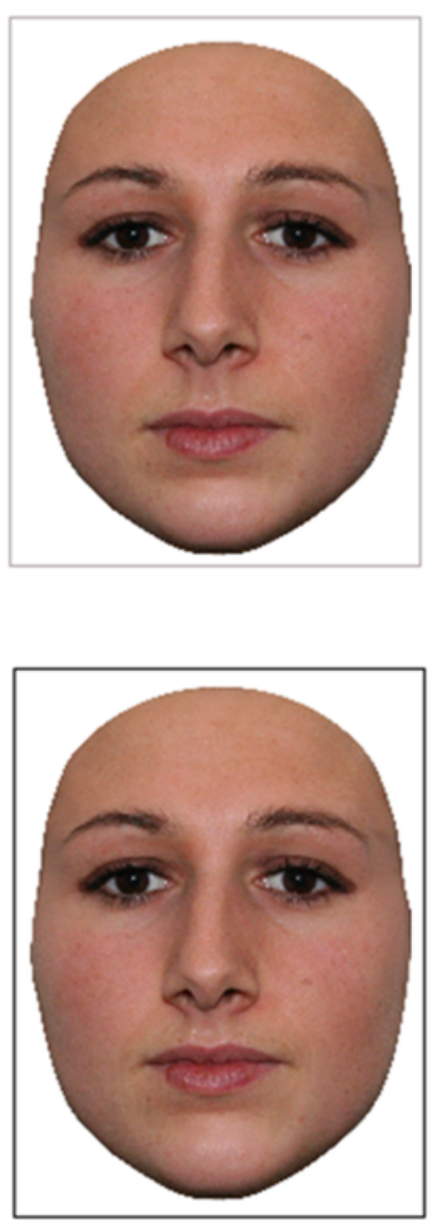
2

Results for Experiment 1: veridicality decision task.

Results for Experiment 1: veridicality decision task. a. Number of errors and normalized RTs (calculated by dividing the mean RT per condition by the mean across conditions) for more and less distorting conditions (bars represent standard errors). Single subjects' ranked differences in $\mathbf{b}$. number of errors and $\mathbf{c}$. RTs between conditions.
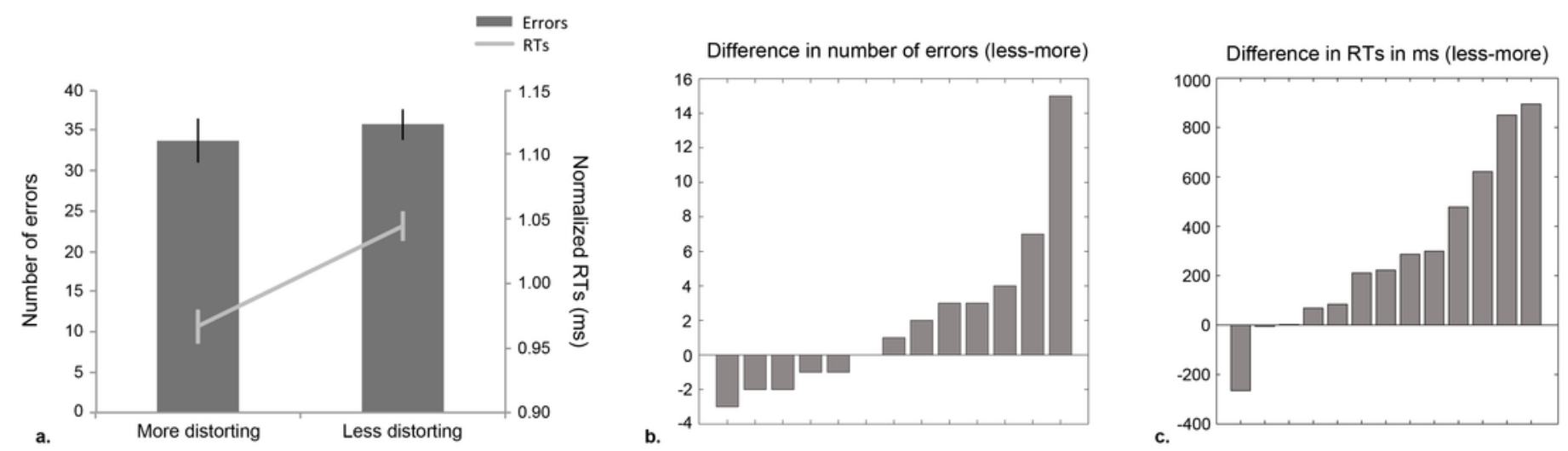


\section{3}

Results for Experiment 2.

Results for Experiment 2. a. Mean performance per group for each of the three trial types presented: trials of interest considered for analyses (original paired with altered facial configurations), and catch trials (stimulus repetition or stimuli depicting different alterations of the facial configuration; see Methods). Performance correct (as opposed to number of errors) is displayed because the total number of trials involving stimulus repetition was equal to the sum of those requiring "different" responses (original/altered, altered/altered). Note that control subjects' performance for trials of interest did not differ from chance level. $\mathbf{b}$. Familiar and unfamiliar subjects' discrimination performance for trials of interest, separately for more and less distorting changes of the overall facial configuration (bars represent standard errors), which were not associated with significant differences in performance. Single subjects' ranked differences in $\mathbf{c}$. number of errors and $\mathbf{d}$. RTs between conditions. 
Group-dependent average performance across all trial types

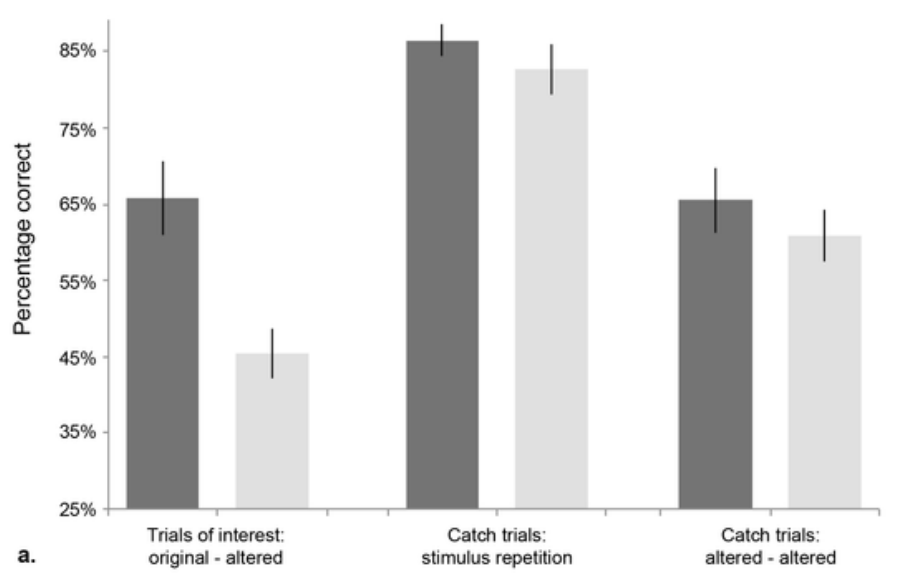

Group-dependent performance for trials of interst: more vs. less distorting changes

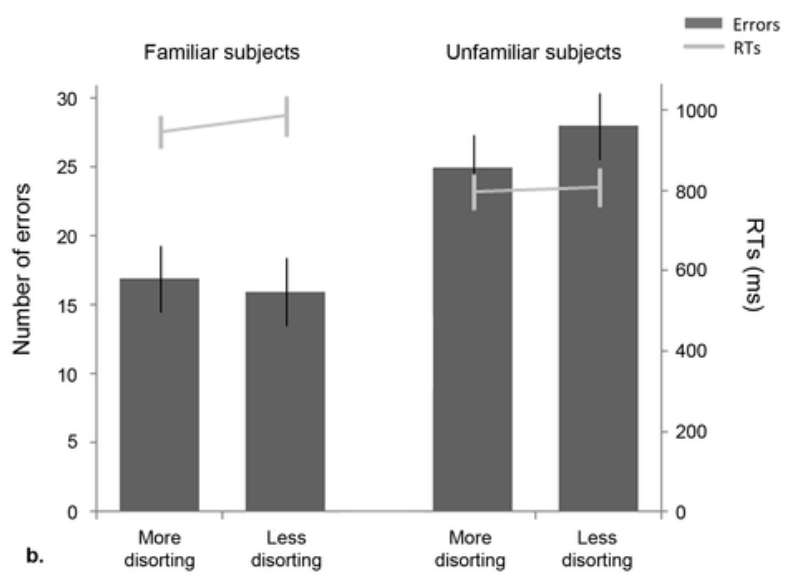

Familiar subjects behavior for trials of interest
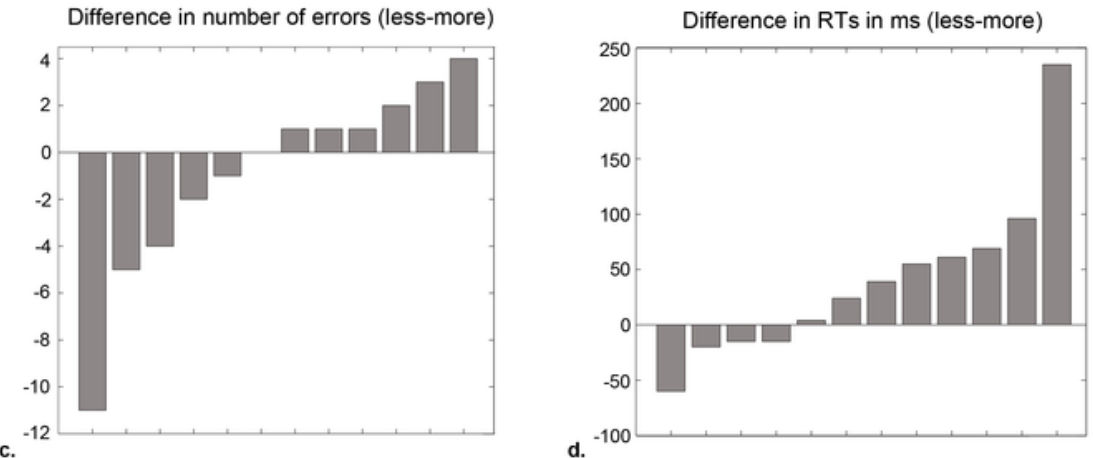

d. 


\section{4}

Personally familiar face identification across simulated viewing distances.

\section{Personally familiar face identification across simulated viewing distances. Mean}

performance and $95 \%$ confidence intervals for identification of colleagues' faces $(N=11$;

mean age: $32 \pm 7$; seven female) plotted as a function of viewing distance. The reduced-size images displayed in the bottom row depict stimuli used in the experiment to simulate viewing distances; the top row displays the visual information projected to the retina. Subjects verbally identified gray scaled, full frontal, naturalistic images of 39 members of the Department of Psychology, University of Glasgow, which were taken under identical viewing conditions and matched for luminosity. Faces were centered on a 1024x1024 pixel canvas with grey background; increasing physical distance between participants and faces to be identified was simulated by shrinking image size with the Laplacian pyramid (Burt \& Adelson, 1983). This recursively removes the highest SFs of an image while down-sampling the residual image by a corresponding amount as done e.g. by Smith \& Schyns (2009). The simulated viewing distances ranged from 3.3 to 844.8 meters, with stimuli recursively downsampled from an initial on-screen size of $24 \mathrm{~cm}$ in height displayed at a $3.3 \mathrm{~m}$ viewing distance (see Loftus \& Harley, 2004). 


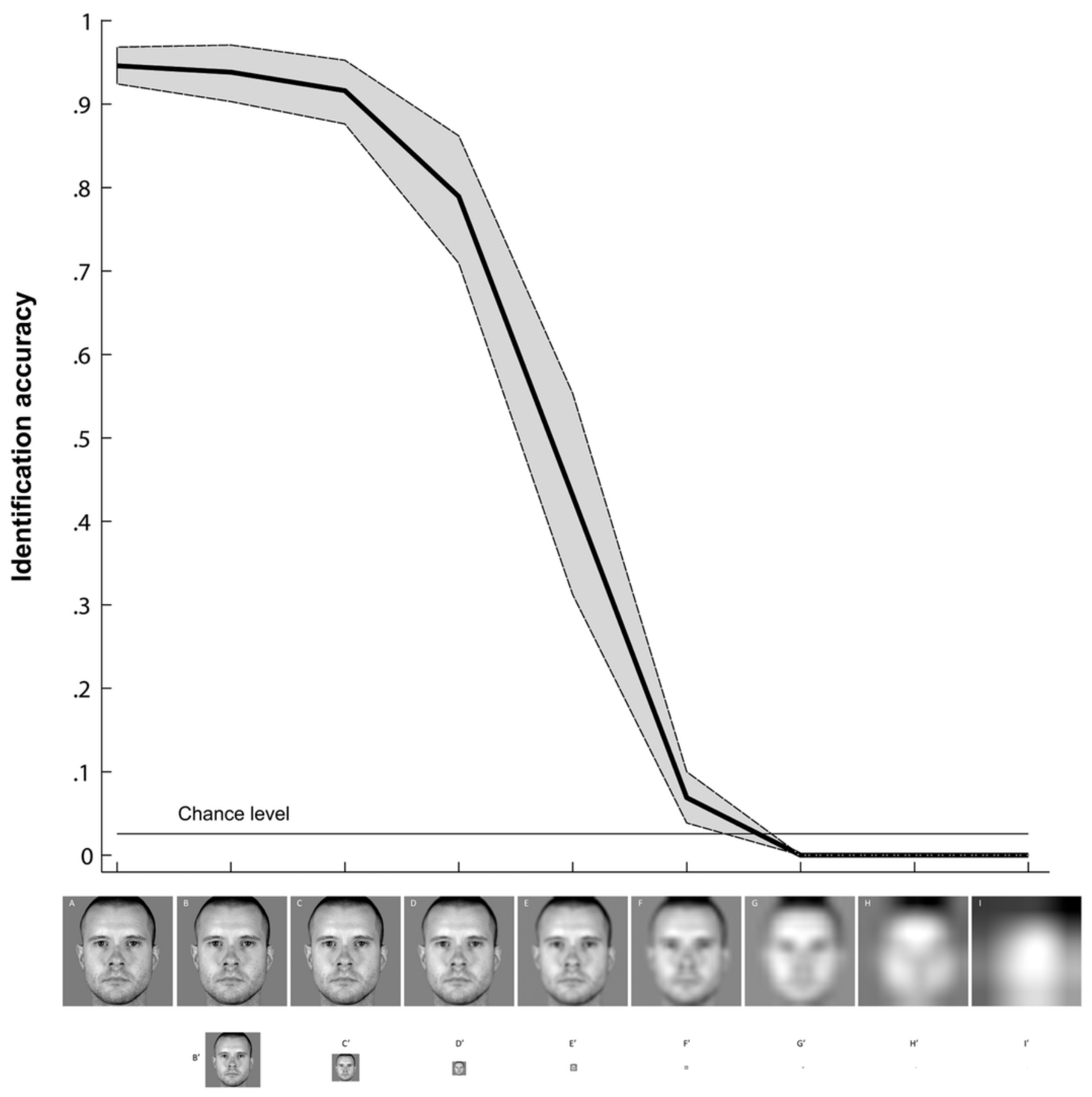

